$03,09,12$

\title{
Поляритонные моды в цилиндрическом микрорезонаторе в режим поляритонного лазера
}

\author{
(С) Л.В. Котова ${ }^{1}$, P.G. Savvidis ${ }^{2,3}$, L. Besombes ${ }^{4}$, В.П. Кочерешко ${ }^{1}$ \\ ${ }^{1}$ Физико-технический институт им. А.Ф. Иофрфе РАН, \\ Санкт-Петербург, Россия \\ ${ }^{2}$ Westlake University, \\ 18 Shilongshan Rd, Hangzhou 310024, Zhejiang, China \\ ${ }^{3}$ Westlake Institute for Advanced Study, \\ 18 Shilongshan Rd, Hangzhou 310024, \\ Zhejiang, China \\ ${ }^{4}$ Institut Néel, CNRS, avenue des Martyrs BP 166, \\ Fr-38042 Grenoble Cedex 9, France \\ E-mail: kotova@mail.ioffe.ru
}

Поступила в Редакцию 1 января 2021 г.

В окончательной редакции 1 января 2021 г.

Принята к публикации 9 января 2021 г.

\begin{abstract}
Исследованы спектры экситон-поляритонной фотолюминесценции из микрорезонаторов цилиндрической формы при оптическом возбуждении ниже и выше порога перехода в режим поляритонного лазера в условиях сильной экситон фотонной связи. При относительно слабом оптическом возбуждении проявлялись моды латерального квантования поляритонов и моды шепчущей галереи. Наблюдалось как спектральное распределения этих мод, так и пространственная зависимость их волновых функций в плоскости резонатора. С увеличением интенсивности возбуждения и переходе в режим поляритонного лазера в спектре оставалась только одна, самая длинноволновая линия лазерной генерации. Высказано предположение о том, что при сильном оптическом возбуждении в спектре присутствует не только экситон-поляритонное излучение, но и трион-поляритонное.
\end{abstract}

Ключевые слова: экситоны, поляритоны, полупроводники, оптика, микрорезонаторы.

DOI: 10.21883/FTT.2021.05.50809.001

\section{1. Введение}

Микрорезонаторы широко используются для изготовления вертикальных поверхностно излучающих лазеров (VCSEL) [1]. VCSEL имеют ряд преимуществ по сравнению с „обычными“ лазерами с излучением из торца.

1. Поскольку VCSEL излучают с верхней поверхности, то в процессе производства их можно проверять, еще до того, как они будут разделены на отдельные устройства. Это существенно уменьшает выход негодных и снижает стоимость конечного продукта.

2. Возможно создавать двухмерные массивы VCSEL, матрицы из лазеров - лазерные экраны.

3. Большая выходная апертура VCSEL обеспечивает меньший угол расходимости выходного луча и обеспечивает высокую эффективность связи с оптическими волокнами.

4. Небольшой размер активной области по сравнению с лазерами с торцевым излучением снижает пороговый ток VCSEL, что приводит к низкому энергопотреблению [2].

Микрорезонаторы служат основой для создания поляритонных лазеров [3]. В таких лазерах не используется эффект инверсной заселенности для усиления света и вынужденного излучения, явления, которое лежит в основе работы всех лазеров и давшего лазерам их название. С другой стороны, так же, как и все лазеры, поляритонный лазер является источником когерентного монохроматического света. Основным преимуществом поляритонных лазеров по сравнению с обычными является их крайне низкое энергопотребление.

Процесс создания инверсной заселенности в обычных лазерах энергетически не эффективен. Из принципа Паули следует, что число электронов, которые одновременно могут находиться на заданном энергетическом уровне, ограничено. В результате, обычные лазеры расходуют энергию на то, чтобы „забросить“ электроны на более высокие энергетические уровни.

В поляритонном лазере электроны соединяются с имеющимися в полупроводнике дырками, образуя экситоны. Экситоны являются бозонами и могут находиться на любом энергетическом уровне в неограниченном количестве. Экситон, взаимодействуя с фотоном, превращается в поляритон, который достигнув поверхности излучает фотон, идентичный исходному. Использование бозонов позволяет существенно снизить порог, на котором может работать лазер. В результате поляритонные лазеры потребляют в сотни раз меньше энергии, чем традиционные, при тех же параметрах излучения. Поляритонный лазер может оказаться незаменимым для создания квантовых компьютеров [4]. 
Важнейшим элементом любого лазера, как обычного, так и поляритонного, является оптический резонатор. Все лазеры излучают на частотах, соответствующих собственным модам этого резонатора.

Помимо обычных продольных мод в резонаторах могут существовать и поперечные оптические моды. Такие моды особенно важны для резонаторов малого размера - микрорезонаторов.

Среди таких поперечных мод важнейшее значение имеют так называемые моды шепчущей галереи [5]. Эти стоячие волны испытывают полное внутреннее отражение от боковых стенок резонатора и как бы „прижаты“ к ним. В результате такие моды имеют очень высокую добротность [6] и могут сильно влиять на работу лазеров, как VECSEL так и поляритонного [7]. Однако вывод излучения из таких мод и расходимость пучка совершенно неудовлетворительные [8].

В настоящей работе изучались спектры излучения мод латерального квантования поляритонов в цилиндрическом микрорезонаторе диаметром $14 \mu \mathrm{m}$ как в линейном, так и в нелинейном режиме при оптическом возбуждении при температуре $5 \mathrm{~K}$. Установлено, что в линейном режиме наблюдаются все латеральные моды и моды шепчущей галереи, а при переходе к режиму лазерной генерации поляритонного лазера - только одна из мод, самая нижняя по энергии. С ростом интенсивности оптического возбуждения эта линия заметно уширялась, что выглядит странно, в отсутствии непрерывного спектра энергий поляритонов.

\section{2. Эксперимент}

Исследуемые структуры представляли собой круглые мезаструктуры (далее меза) диаметром от 1 до $40 \mu \mathrm{m}$, изготовленные из микрорезонатора. Микрорезонатор состоял из микрополости $\mathrm{Al}_{0.25} \mathrm{Ga}_{0.75} \mathrm{As}$ толщиной $5 \lambda / 2$ окруженной двумя брэгговскими зеркалами, состоящими из 35 пар слоев AlAs и $\mathrm{Al}_{0.15} \mathrm{Ga}_{0.85} \mathrm{As}$. Добротность резонатора была равна 20000. Внутрь микрополости, в пучности стоячей электромагнитной волны были помещены 4 квантовые ямы GaAs для того, чтобы усилить взаимодействие экситонов со светом (рис. 1). Ширина ям была выбрана такой, чтобы имел место резонанс между фотонной модой и основным состоянием экситона в ямах, то есть режим сильной экситон - фотонной связи.

Регистрировались спектры фотолюминесценции (ФЛ) от этих структур при температуре $5 \mathrm{~K}$. Для эффективного возбуждения ФЛ использовалось лазерное излучение с энергией кванта $1.62 \mathrm{eV}$ (7652 A), что соответствует области выше „стоп-зоны“ но ниже поглощения в брэгговских зеркалах, диаметр пятна на поверхности образца был $\sim 2 \mu \mathrm{m}$. Для регистрации сигнала ФЛ использовалась установка микрофотолюминесценции, которая состояла их микрообъектива, монохроматора и ССД камеры.

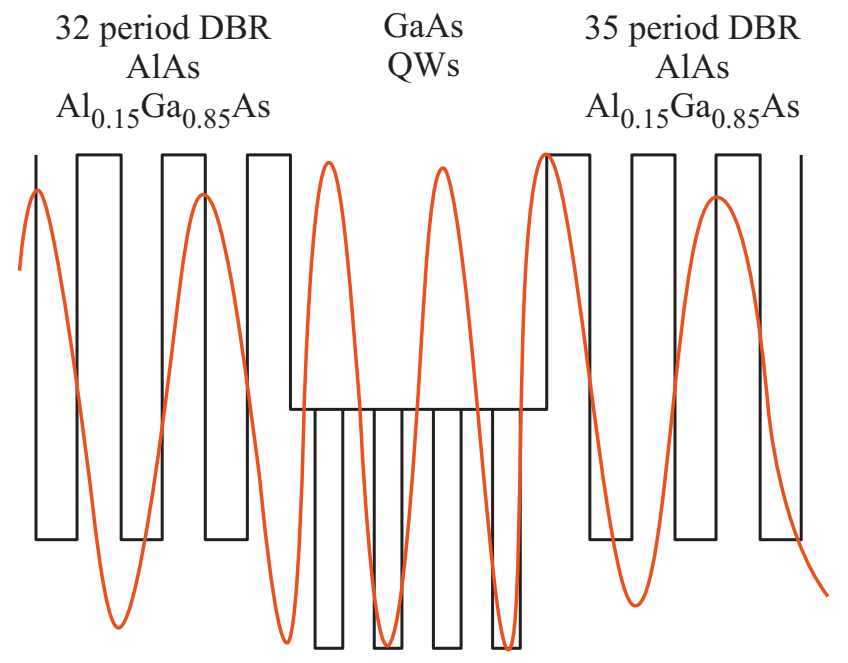

Рис. 1. Зонная схема исследуемой структуры.

При достаточно слабом оптическом возбуждении (меньше $0.3 \mathrm{~mW}$ ) в спектре ФЛ мезы диаметром $14 \mu \mathrm{m}$, в диапазоне от 1.5405 до $1.5445 \mathrm{eV}$ наблюдается широкая бесструктурная полоса излучения. Отсутствие структуры этой полосы обусловлено эффективным заселением состояний всей поляритонной зоны при нерезонансном фото-возбуждении. При достаточно больших потерях, связанных экситонным затуханием, в мезах большого диаметра (десятки микрон), стоячие волны не могут сформироваться, поэтому в спектре излучения поляритонов не наблюдается никакой структуры.

С увеличением интенсивности оптического возбуждения, когда „приход“ экситонов начинает превосходить их потери из-за затухания, в спектре поляритонной ФЛ появляется структура, связанная с размерным квантованием экситонных поляритонов в плоскости мезы (рис. 2, $a$ ).

При еще большей интенсивности возбуждения и переходе в режим поляритонного лазера, эти линии также быстро насыщаются и пропадают из спектра, и в спектре остается только одна самая длинноволновая линия (рис. 2, $b$ ). Этот режим по сути дела соответствует режиму бозе конденсации экситонных поляритонов [3]. При дальнейшем росте интенсивности оптического возбуждения эта линия уширяется в сторону больших энергий, и ее ширина достигает нескольких $\mathrm{meV}$ при достаточно интенсивном возбуждении (рис. 2,c).

Благодаря использованию микро-ФЛ, вдобавок к спектральному распределению мод размерного квантования поляритонов нам удалось зарегистрировать и пространственную зависимость интенсивности излучения из этих мод, прямо отражающую пространственное изменение их волновых функций.

На рис. 3, $a$ представлен спектр поляритонной фотолюминесценции. По горизонтальной шкале представлены энергии и длины волн, а по вертикальной шкале 

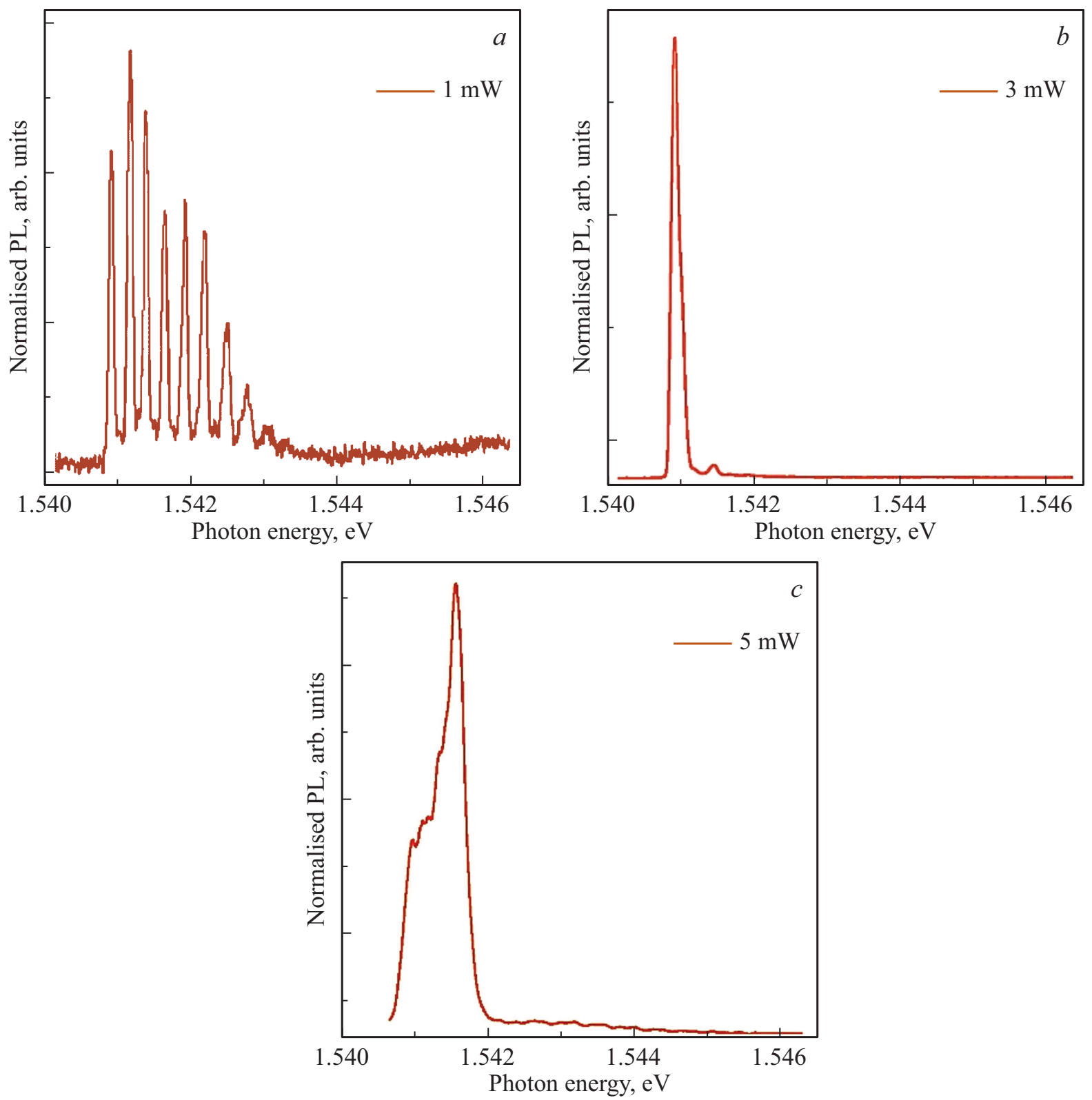

Рис. 2. $a$-- спектр фотолюминесценции мезы диаметром $14 \mu \mathrm{m}$ при относительно слабом оптическом возбуждении, нормированный на максимальную интенсивность. $b-$ спектр излучения при среднем возбуждении выше порога перехода к лазерной генерации поляритонного лазера. $c$ - спектр излучения при интенсивном оптическом возбуждении.

пространственная координата, интенсивность сигнала представлена цветом в логарифмическом масштабе. На рис. $3, b$ представлено пространственное распределение полной, интегральной интенсивности ФЛ. Излучение длинноволновых мод квантования поляритонов сосредоточено ближе к центру мезы, а коротковолновых мод ближе к краям. Самые коротковолновые моды „прижаты“ к краю мезы. Это - моды шепчущей галереи.

На рис. 3 можно увидеть и пространственное распределение плотности экситонных поляритонов на уровнях размерного квантования. Так для низких по энергии уровней, плотность поляритонов имеет максимум в центре мезы, а для более высоких по энергии уровней, имеется несколько максимумов плотности.

\section{3. Теория}

В условиях резонанса между экситонной и фотонной модами в микрорезонаторе экситоны и фотоны перестают быть независимыми друг от друга и формируют смешанные состояния - поляритоны. Для описания таких смешанных состояний нужно совместно решить уравнение движения для экситонной поляризации, вызванной электромагнитным полем и уравнение движения 


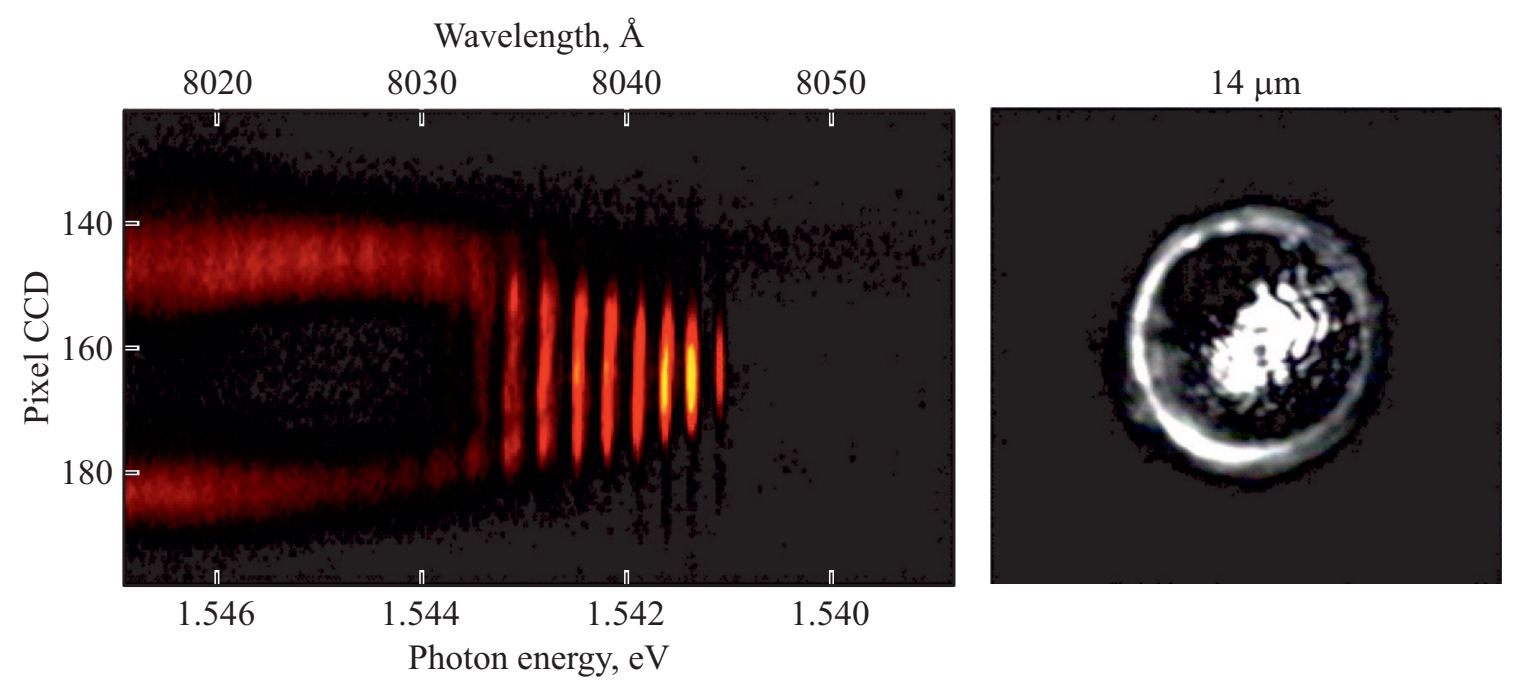

Рис. 3. $a$ - моды латерального квантования поляритонов и шепчущей галереи. Интенсивность линий показана цветом в логарифмическом масштабе. $b$ - интегральная фотолюминесценция мезы при оптическом возбуждении в центр.

для электромагнитного поля, вызванного экситонной поляризацией.

Уравнение для поляризации $\mathbf{P}_{\text {exc }}$, связанной с возбуждением экситона, имеет вид [9]:

$$
\left[\hat{H}_{i j}(\mathbf{K})-\hbar \omega \delta_{i j}\right] P_{i}^{\mathrm{exc}}(\omega, \mathbf{K})=d_{i}(\mathbf{K}) \cdot d_{j}^{*}(\mathbf{K}) \cdot E_{j}(\omega, \mathbf{K}) .
$$

Здесь $d-$ матричный элемент дипольного момента экситонного перехода. Уравнение движения электромагнитного поля под действием экситонной поляризации записывается в виде

$$
\begin{gathered}
\Delta \mathbf{E}(\mathbf{r}, t)-\operatorname{grad}(\operatorname{div} \mathbf{E}(\mathbf{r}, t))=\frac{1}{c^{2}} \frac{\partial^{2}}{\partial t^{2}} \mathbf{D}(\mathbf{r}, t), \\
\mathbf{D}=\varepsilon \mathbf{E}+4 \pi \mathbf{P} .
\end{gathered}
$$

Систему дифференциальных уравнений (1),(2) нужно решать совместно.

Для планарной структуры можно пренебречь вторым слагаемым в (2). Хотя наша структура и не вполне планарная, но диаметр мезы гораздо больше толщины резонатора и краевыми эффектами можно пренебречь. Тогда

$$
\Delta \mathbf{E}(\mathbf{r}, \omega)-k^{2} \mathbf{E}(\mathbf{r}, \omega)=4 \pi k_{0}^{2} \mathbf{P}(\mathbf{r}, \omega) .
$$

Уравнение для экситонной поляризации также можно переписать в виде неоднородного уравнения Гельмгольца

$$
\Delta \mathbf{P}(\mathbf{r}, \omega)-q^{2}(\omega) \mathbf{P}(\mathbf{r}, \omega)=d^{2} \mathbf{E}(\omega, \mathbf{r}) .
$$

Здесь $q^{2}(\omega)=-\frac{2 M}{\hbar}\left(\omega_{0}-\omega-i \Gamma\right), \omega_{0}-$ резонансная частота экситона, $\Gamma$ - экситонное затухание, $M-$ трансляционная масса экситона.

Условие разрешимости системы уравнений (3)-(4) приводит к дисперсионному уравнению [10]:

$$
\left(\omega_{\mathrm{exc}}(k)-\omega-i \Gamma\right)\left(\omega_{\mathrm{ph}}(k)-\omega-i \gamma\right)=V^{2} .
$$

Здесь $\hbar \omega_{\mathrm{exc}}(k)=\hbar \omega_{\mathrm{exc}}^{0}+\frac{\hbar^{2} k^{2}}{2 M}-$ энергия экситона, $\hbar \omega_{\mathrm{ph}}(k)=\hbar \omega_{\mathrm{ph}}^{0}+c \hbar k-$ энергия фотонной моды, $c-$ скорость света в вакууме, $\gamma$ - затухание фотонной моды, $V$ - параметр характеризующий взаимодействия экситонной и фотонной мод.

В условиях резонанса резонансные частоты экситона и фотона одинаковы $\omega_{\mathrm{exc}}^{0}=\omega_{\mathrm{ph}}^{0}=\omega_{0}$. Тогда

$$
\omega_{1,2}=\omega_{0}+\frac{\omega_{M}(k)}{2} \pm \frac{1}{2} \sqrt{\left[\omega_{M}(k)\right]^{2}-4 d_{1} d_{2}}
$$

Здесь $\omega_{M}(k)=c \sqrt{k_{\rho}^{2}+k_{\perp}^{2}}=c \sqrt{k_{\rho}^{2}+\left(\frac{5 \pi}{L}\right)^{2}}$ мы считали, что дисперсия экситона мала в интересующем нас масштабе волновых векторов.

Волновые функции поляритонов в цилиндрическом резонаторе описываются так [10]:

$$
\Psi(\rho, \varphi, z) \sim J_{m}\left(\rho \sqrt{k_{\rho}^{2}+k_{\perp}^{2}}\right) \sin \left(k_{\perp} z\right) e^{ \pm i m \varphi} .
$$

Здесь $J_{m}(k \rho)-$ функции Бесселя, $z-$ координата вдоль оси цилиндра, $\varphi$ - угол в плоскости резонатора, $\rho-$ координата вдоль радиуса резонатора, $k_{\perp}-$ волновой вектор вдоль оси $z, m$ - номер функции Бесселя, $n-$ показатель преломления, $k_{0}=\omega / c-$ волновой вектор света в вакууме.

Для нахождения собственных мод необходимо задать граничные условия. Граничное условие общего вида требует равенства нулю линейной комбинации функции и ее градиента по нормали к поверхности. Для простоты будем считать, что собственным модам соответствуют решения, когда $\Psi(\rho, \varphi, z)=0$ на поверхности. Это не должно сильно повлиять на результат, но зато сильно упростит вычисления. Из граничных условий следуют условия на квантовые числа $N, k_{\perp}$ и $m$. 
Квантование по оси $z$ подчиняется условиям $k_{\perp}^{N} L=\pi N, L=\frac{5}{2} \frac{\lambda_{0}}{n}-$ толщина активной области резонатора, $\left(n=3.5, \lambda_{0}=804.5 \mathrm{~nm}\right)$, а квантование в плоскости структуры - условию: $k_{\rho}^{m} R=b_{l, m}, b_{l, m}$ - корни функций Бесселя: $J_{m}\left(b_{l, m}\right)=0, l-$ номер корня, $R-$ радиус мезы. (Будем учитывать только первые корни из каждой функции Бесселя $J_{m}\left(b_{l, m}\right)$, так как из-за наличия механической (экситонной) части поляризации, поляритоны быстро релаксируют по энергии).

Отсюда находим все поляритонные моды латерального квантования поляритонов в нашем резонаторе, они обозначены на рис. 2.

\section{4. Обсуждение результатов}

В спектрах фотолюминесценции цилиндрических микрорезонаторов в режиме сильной экситон-фотонной связи при слабом оптическом возбуждении наблюдалась широкая, бесструктурная полоса излучения. Отсутствие структуры этой полосы обусловлено достаточно большими потерями в экситонной и фотонной модах из-за экситонного затухания и ухода излучения через боковые стенки мезы. Высокие потери приводят к тому, что стоячие поляритонные волны не могут сформироваться, и в структуре достаточно большого размера, наблюдается широкая полоса излучения отражающая заселенность всей экситон-поляритонной зоны.

При увеличении интенсивности оптического возбуждения в спектре проявляются узкие линии ФЛ, соответствующие модам латерального квантования поляритонов. Число этих мод и энергетическое расстояние между ними определяется дисперсией поляритонов и размером резонатора. Спектральное положение пиков ФЛ определяется условием квантования $k_{\rho}^{m} R=b_{l, m}$, где $b_{l, m}-$ корни функций Бесселя.

Благодаря использованию микрофотолюминесценции удалось визуализировать спектральное положение и пространственное распределение интенсивности всех этих мод. На рис. 3 слева видно, что интенсивность этих пиков меняется по ширине мезы. Эта модуляция связана с пространственной зависимостью волновой функции поляритона (7). Помимо мод латерального квантования поляритонов, нами наблюдались и поляритонные моды шепчущей галереи, как бы прижатые к краю мезы (рис. 3).

При увеличении интенсивности возбуждения выше некоторого порога в спектре остается одна самая низкая по энергии поляритонная моды, а остальные моды подавляются. Это соответствует по сути дела режиму Бозе-конденсации, когда все поляритоны собираются на самом нижнем энергетическом уровне.

Вызывает удивление то, что с ростом интенсивности возбуждения происходит уширение этой линии. Это выглядит необычно, так как наша меза представляет собой квантовую точку для поляритонов, в которой поляритоны квантуются по всем трем направлениям.
В этом случае сплошной спектр поляритонов отсутствует, и могут наблюдаться только очень узкие линии их размерного квантования.

Наблюдаемое поведение спектра излучения поляритонного лазера может быть связано с тем, что при нерезонансном оптическом возбуждении рождаются не только экситоны, но и не связанные электрон-дырочные пары. Дырки обычно быстро захватываются на примеси, и в структуре всегда присутствует некоторое количество свободных электронов. Взаимодействуя с экситонами, электроны могут образовывать трионы [11]. В результате в структуре будут существовать не только экситонны, но и трионы.

Сила осциллятора триона сравнима с силой осциллятора экситона [12], и в резонаторе трионы могут формировать трионные поляритоны аналогичные экситонным. Их резонансные энергии и расщепление Раби должны быть очень близки друг к другу.

Ширина трионной линии зависит от концентрации электронов и, следовательно, должна расти с ростом интенсивности оптического возбуждения.

\section{5. Выводы}

В настоящей работе исследованы спектры поляритонной фотолюминесценции из микрорезонаторов цилиндрической формы при оптическом возбуждении ниже и выше порога перехода в нелинейный режим в условиях сильной экситон фотонной связи. В спектрах наблюдались яркие моды латерального квантования экситонных поляритонов с малым орбитальным квантовым числом $m<10$, в резонаторах относительно большого диаметра, наблюдались экситон-поляритонные моды шепчущей галереи с большими $m>10$.

Благодаря использованию микро-ФЛ, в добавок к спектральному распределению мод размерного квантования поляритонов нам удалось зарегистрировать и пространственную зависимость волновой функции поляритона в резонаторе [см. уравнение (7)].

В режиме „поляритонного лазера“ „выживала“ только одна мода латерального квантования поляритонов с минимальной энергией. Остальные моды и моды шепчущей галереи, несмотря на более высокую добротность, не участвуют в генерации.

С ростом интенсивности оптического возбуждения линия излучения поляритонного лазера уширялась. Это может быть связано с рождением трионных поляритонов.

\section{Финансирование работы}

K.V.P. и K.L.V. благодарят грант РФФИ № 19-02-00237. S.P.G. благодарит the Westlake University Foundation and the program 2018R01002 supported by Leading Innovative and Entrepreneur Team Introduction Program of Zhejiang. 


\section{Конфликт интересов}

Авторы заявляют, что у них отсутствует конфликт интересов.

\section{Список литературы}

[1] F. Koyama, S. Kinoshita, K. Iga. Trans. IEICE E71, 1089 (1988).

[2] V. Jayaraman, J. Jiang, B. Potsaid, G. Cole, J. Fujimoto, A. Cable. Proc. SPIE 8276, 82760D, (2012).

[3] A. Imamoglu, R.I. Ram, S. Pan, Y. Tamamoto. Phys. Rev. A53, 4250 (1996).

[4] M.T. Hill, M.C. Gather. Nature Photon. 8, 908 (2014).

[5] G.C Righini, Y. Dumeige, P. Feron, M. Ferrary, G. Nunzi Cinti, D. Ristic, S. Soria. Nuovo Cimento 34, 2041 (2011).

[6] Y.X. Jiang, L. Shao, Shu-Xin Zhang, X. Yi, J. Wiersing, L. Wang, Q. Grang, M. Loncar, L. Yang, Yu-Fang Xiao. Science 358, 344 (2017).

[7] E. Gornik. Science 280, 1544 (1998).

[8] C.G. Garrett, W. Kaiser, W.L. Bond. Phys. Rev. 124, 1807 (1961).

[9] E.L. Ivchenko. Excitons. In: North-Holland Publishing Company (1982). V. 2. P. 865.

[10] A.V. Kavokin, J.J. Baumberg, G. Malpuech, F.P. Laussy. Microcavities. Oxford University Press (2006). P. 468.

[11] G. Finkelstein, H. Shtrikman, I. Bar-Joseph. Phys. Rev. Lett. 74, 976 (1995).

[12] G.V. Astakhov, V.P. Kochereshko, D.R. Yakovlev, W. Ossau, J. Nrurnberger, W. Faschinger, G. Landwehr. Phys. Rev. B 62, 10345 (2000).

Редактор Ю.Э. Китаев 